\title{
Exosomal microRNA profiles from serum and cerebrospinal fluid in neurosyphilis
}

\author{
Huan Chen, Yuan Zhou, Zhao-Yuan Wang, Bing-Xi Yan, Wei-Fang Zhou, \\ Ting-Ting Wang, Min Zheng, Xiao-Yong Man ${ }^{\odot}$
}

- Additional material is published online only. To view please visit the journal online (http://dx.doi.org/10.1136/ sextrans-2018-053813)

Department of Dermatology, Zhejiang University School of Medicine, Second Affiliated Hospital, Hangzhou, China

\section{Correspondence to} Professor Xiao-Yong Man, Department of Dermatology, Zhejiang University School of Medicine, Second Affiliated Hospital, Hangzhou 310009, China; manxy@zju.edu.cn

$\mathrm{HC}$ and $\mathrm{YZ}$ contributed equally.

Received 20 August 2018 Revised 14 February 2019

Accepted 3 March 2019 Published Online First 29 March 2019
Check for updates

(C) Author(s) (or their employer(s)) 2019. No commercial re-use. See rights and permissions. Published by BMJ.

To cite: Chen $\mathrm{H}$

Zhou Y, Wang Z-Y,

et al. Sex Transm Infect

2019:95:246-250.

\begin{abstract}
Objective Changes in microRNAs (miRNAs) in the cerebrospinal fluid (CSF) are associated with different neurological diseases. Since alternations of miRNAs in neurosyphilis are insufficiently investigated, we analysed miRNAs in the CSF of patients suffering from neurosyphilis.
\end{abstract}

Methods Exosomes were isolated from serum and CSF. Levels of 44 miRNAs were determined using quantitative real-time PCR-based miRNA array.

Results In patients with neurosyphilis (NSP), miR-590$5 p$, miR-570-3p and miR-570-5p were upregulated in the CSF and serum, when compared with patients with syphilis without neurosyphilis (SP). miR-590-5p and miR-570-3p were significantly upregulated $(p<0.001)$. The expression of miR-21-5p was upregulated only in the CSF of NSP. Significant downregulation was observed for miR-93-3p in the CSF and serum of NSP. No statistical difference was found in the expression of miR-7-5p, miR-1307-5p, miR-203a-3p, miR-16, miR-23b-3p and miR-27b-5p in the CSF and serum of NSP and SP. Conclusion For the first time, regulation profiles in miRNA in the CSF and serum were analysed in NSP. We found significant differences in upregulation and downregulation. Therefore, miRNAs may be potential biomarkers for the presence of neurosyphilis.

\section{INTRODUCTION}

Syphilis, a disease that shows increasing incidence, is caused by the bacterium Treponema pallidum. $1 \mathrm{In}$ the course of the disease, approximately one-third of patients will develop a neuronal infestation, a neurosyphilis. Particularly at risk are patients with decreased immune system, such as HIV-positive patients ${ }^{1}$ and individuals who were not sufficiently treated with antibiotics.

T. pallidum cannot be cultured in vitro. To date, there has been a lack of a highly sensitive and specific diagnostic test. Therefore, the diagnosis of neurosyphilis relies on clinical findings and on cerebrospinal fluid (CSF) abnormalities. ${ }^{1}$ Because the sampling of CSF is challenging due to its invasive manner, it is of importance to find a sensitive and easy-to-implement test.

Exosomes represent a specific subtype of secreted membrane nanovesicles that are approximately 30-100 $\mathrm{nm}$ in size and are formed inside the secreting cells in endosomal compartments, called multivesicular bodies. Biofluids such as plasma/ serum, urine, breast milk and CSF are potential

\section{Key messages}

- Comparing patients with and without neurosyphilis, patients with neurosyphilis showed a significant upregulation in miR590-5p, miR-570-3p and miR-570-5p in the cerebrospinal fluid and serum.

- A significant downregulation was measured for miR-93-3p.

- These microRNAs may be potential biomarkers for the presence of neuronal infestations in patients with syphilis.

sources for exosomes. ${ }^{2} 3$ These secreted vesicles are known to play an important role in normal physiological processes and also may be exploited in pathological conditions such as viral infections, making them a possible pool for detection of novel biomarkers. $^{2} 3$ Exosomes contain a lipid bilayer which is known to encapsulate proteins, microRNAs (miRNAs) and messenger RNA (mRNAs), which protects them from degradation. Due to their low immunity, good transmission and ability to cross the blood-brain barrier (BBB), exosomes are used for therapeutic delivery systems effectively. ${ }^{4-7}$

Brain function depends on the interaction between the neurons and the glial cells. Recent evidence suggests that the exosomes released by these cells play an important role in neuroglial transmission. ${ }^{8}$ Changes of miRNAs in exosomes have been reported for the diagnosis of different diseases, as well as a potential biological marker for brain neoplasms, degenerative diseases, autism and schizophrenia. ${ }^{9}$ In the meantime, these changes in miRNAs, as biological markers of diseases in the central nervous system (CNS), can be detected in a patient's serum. ${ }^{10} 11$ Research on alternative miRNAs associated with neurodiseases transmission through BBB is in progress. ${ }^{11} 12$

Profiling of blood cells, exosomes or body fluid miRNAs would represent a tremendous and promising advance in non-invasive diagnostics for CNS disorders. ${ }^{9}$ There has been a report of miRNA changes in CSF associated with type B encephalitis infection. ${ }^{12}$

The question arises whether CSF-based miRNAs could be a useful biomarker for the detection of neurosyphilis. For this we examined the expression pattern of 44 miRNAs in the CSF of patients with syphilis with and without involvement of CNS. 
Table 1 Clinical characteristics of the 34 patients

\begin{tabular}{|c|c|c|c|c|c|c|c|c|c|c|c|}
\hline \multirow{2}{*}{$\begin{array}{l}\text { Patient } \\
\text { number }\end{array}$} & \multirow[b]{2}{*}{ Sex } & \multirow[b]{2}{*}{ Age } & \multirow{2}{*}{$\begin{array}{l}\text { Confirmation } \\
\text { time }\end{array}$} & \multirow[b]{2}{*}{ Treatment history } & \multirow{2}{*}{$\begin{array}{l}\text { Serum } \\
\text { RPR titre }\end{array}$} & \multirow{2}{*}{$\begin{array}{l}\text { Serum TPPA } \\
\text { titre }\end{array}$} & \multirow{2}{*}{$\begin{array}{l}\text { CSF RPR } \\
\text { titre }\end{array}$} & \multirow{2}{*}{$\begin{array}{l}\text { CSF TPPA } \\
\text { titre }\end{array}$} & \multicolumn{3}{|c|}{ CSF findings } \\
\hline & & & & & & & & & Glu & Protein & Cell count \\
\hline 1 & $M$ & 58 & 1 year & Untreated & - & + & - & - & 3.92 & 36.90 & 4 \\
\hline 2 & M & 47 & 7 years & Roxithromycin & $1: 1$ & + & - & - & 4.05 & 111.40 & 5 \\
\hline 3 & $\mathrm{~F}$ & 62 & 1.5 years & Benzathine penicillin & $1: 8$ & + & - & - & 3.15 & 34.20 & 6 \\
\hline 4 & $\mathrm{~F}$ & 48 & 2 years & Benzathine penicillin & $1: 1$ & + & - & - & 3.50 & 43.80 & 2 \\
\hline 5 & $\mathrm{~F}$ & 49 & 1 year & Untreated & - & + & - & - & 3.90 & 67.30 & 2 \\
\hline 6 & M & 71 & Unknown & Untreated & - & + & - & - & 3.46 & 62.40 & 2 \\
\hline 7 & $\mathrm{~F}$ & 36 & 5 years & Untreated & $1: 1$ & + & - & - & 3.54 & 16.50 & 2 \\
\hline 8 & M & 49 & 2 years & Eryphilin & $1: 2$ & + & - & - & 3.93 & 28.20 & 4 \\
\hline 9 & $\mathrm{~F}$ & 26 & 3 months & Benzathine penicillin & $1: 1$ & + & - & - & 3.33 & 26.10 & 2 \\
\hline 10 & M & 59 & Unknown & Untreated & $1: 2$ & + & - & - & 3.60 & 28.80 & 4 \\
\hline 11 & $\mathrm{~F}$ & 34 & 3 years & Benzathine penicillin & $1: 16$ & + & - & - & 3.84 & 28.00 & 6 \\
\hline 12 & $\mathrm{~F}$ & 29 & 2 years & Benzathine penicillin & $1: 4$ & + & - & - & 3.45 & 14.20 & 2 \\
\hline 13 & $\mathrm{~F}$ & 43 & 3 years & Untreated & $1: 8$ & + & - & - & 3.84 & 31.50 & 6 \\
\hline 14 & $\mathrm{~F}$ & 41 & 4 years & Benzathine penicillin & 1:1 & + & - & - & 3.19 & 28.40 & 2 \\
\hline 15 & $\mathrm{~F}$ & 77 & Unknown & Untreated & 1:1 & + & - & - & 3.47 & 43.20 & 4 \\
\hline 16 & $\mathrm{~F}$ & 32 & Unknown & Untreated & $1: 8$ & + & - & - & 3.61 & 24.00 & 0 \\
\hline 17 & M & 74 & Unknown & Untreated & - & + & - & - & 3.69 & 36.20 & 7 \\
\hline 18 & $\mathrm{~F}$ & 54 & Unknown & Untreated & $1: 2$ & + & - & - & 3.11 & 77.70 & 48 \\
\hline 19 & $M$ & 53 & Unknown & Untreated & $1: 8$ & + & $1: 1$ & + & 3.83 & 47.50 & 12 \\
\hline 20 & M & 63 & Unknown & Untreated & $1: 32$ & + & $1: 8$ & + & 3.03 & 66.40 & 26 \\
\hline 21 & $M$ & 56 & 7 months & $\begin{array}{l}\text { Aqueous penicillin + } \\
\text { benzathine penicillin }\end{array}$ & $1: 256$ & + & $1: 8$ & + & 4.29 & 54.30 & 2 \\
\hline 22 & M & 42 & Unknown & Untreated & $1: 32$ & + & $1: 4$ & + & 3.20 & 146.40 & 2 \\
\hline 23 & $\mathrm{~F}$ & 63 & 10 months & Benzathine penicillin & $1: 4$ & + & $1: 1$ & + & 4.68 & 41.80 & 2 \\
\hline 24 & $\mathrm{M}$ & 58 & 1 month & Untreated & $1: 32$ & + & $1: 4$ & + & 4.23 & 47.60 & 116 \\
\hline 25 & M & 41 & Unknown & Untreated & $1: 16$ & + & $1: 8$ & + & 3.59 & 72.30 & 116 \\
\hline 26 & $\mathrm{M}$ & 71 & Unknown & Untreated & $1: 16$ & + & $1: 2$ & + & 3.95 & 45.20 & 24 \\
\hline 27 & $\mathrm{~F}$ & 62 & Unknown & Untreated & $1: 128$ & + & $1: 4$ & + & 2.90 & 75.70 & 50 \\
\hline 28 & $\mathrm{M}$ & 50 & 6 months & Untreated & 1:16 & + & $1: 2$ & + & 3.35 & 28.80 & 20 \\
\hline 29 & $M$ & 44 & Unknown & Untreated & 1:128 & + & $1: 8$ & + & 3.60 & 74.20 & 138 \\
\hline 30 & $\mathrm{~F}$ & 56 & Unknown & Untreated & $1: 32$ & + & 1:8 & + & 2.48 & 100.00 & 40 \\
\hline 31 & $M$ & 41 & Unknown & Untreated & $1: 32$ & + & $1: 4$ & + & 3.02 & 56.60 & 38 \\
\hline 32 & M & 62 & Unknown & Untreated & $1: 64$ & + & $1: 4$ & + & 2.77 & 95.80 & 12 \\
\hline 33 & $M$ & 34 & 1 year & Benzathine penicillin & $1: 8$ & + & $1: 2$ & + & 2.72 & 95.40 & 56 \\
\hline 34 & $M$ & 71 & Unknown & Untreated & $1: 32$ & + & 1:1 & + & 3.46 & 53.60 & 20 \\
\hline
\end{tabular}

CSF, cerebrospinal fluid; F, female; Glu, glucose; M, male; RPR, rapid plasma reagin; TPPA, T pallidum particle agglutination.

\section{MATERIALS AND METHODS}

\section{Serum and CSF sample collection}

Serum and CSF samples were taken from 34 patients with syphilis and neurosyphilis aged 26-77 (table 1) referred to the Department of Dermatology at the Second Affiliated Hospital, Zhejiang University School of Medicine. After obtaining consent, patients were included in our study.

The diagnostic criteria for neurosyphilis that complied with the 2015 guidelines of the Centers for Disease Control and Prevention in America were used to diagnose neurosyphilis (https://stacks.cdc.gov/view/cdc/31403). HIV-infected individuals were excluded.

\section{Exosome isolation}

Six CSF-positive and six CSF-negative samples were randomly chosen. Exosomes were isolated from $500 \mu \mathrm{L}$ of serum and CSF samples using exoRNeasy Serum/Plasma Midi Kit (Exiqon QIAGEN, \#77044) according to the manufacturer's instructions. Briefly, the samples were put onto the exoEasy spin column and centrifuged for $1 \mathrm{~min}$ at $500 \mathrm{~g}$. Then the lysate was collected from the column after QIAzol washing for $5 \mathrm{~min}$ at $5000 \mathrm{~g}$. Then we mixed the lysate and chloroform, shook it vigorously for $15 \mathrm{~s}$, and centrifuged it for $15 \mathrm{~min}$ at 12000 $g$ at $4^{\circ} \mathrm{C}$. After this we collected the upper aqueous phase and mixed it with ethanol. The mix was added into a new RNeasy MinElute Spin Column, centrifuged at 10000 revolutions per minute (rpm) for $15 \mathrm{~s}$ at room temperature (RT). Finally the column was washed with buffer ribulose-5-phosphate-3-epimeras (RPE) for three times. The RNA was collected from the column after RNase-free water washing.

\section{qRT-PCR assay}

The extracted miRNA was reversed into DNA using miScript II RT Kit (Exiqon QIAGEN, \#218161). Quantitative real-time PCR (qRT-PCR) was performed in $20 \mu \mathrm{L}$ reaction mixtures $(0.5$ $\mu \mathrm{M}$ of each primer, $10 \mu \mathrm{L} 2 \times$ SYBR Green Master Mix; Applied Biosystems, Foster City, California, USA) using the following parameters: 40 cycles of $95^{\circ} \mathrm{C}$ for $20 \mathrm{~s}$ and $60^{\circ} \mathrm{C}$ for $40 \mathrm{~s}$. Primers were designed by miRprimer as described ${ }^{13}$ and listed in online supplementary table 1 . 
A

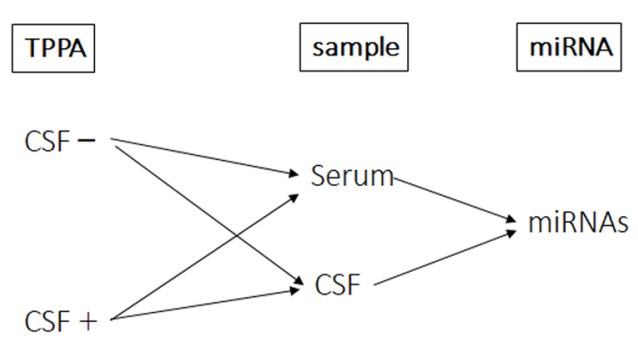

B

miR-210-5p

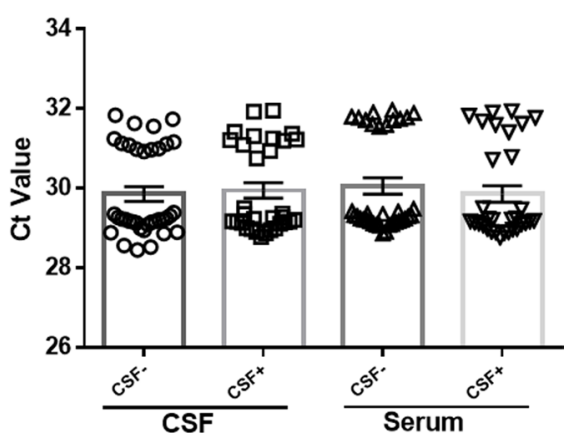

Figure 1 Stable expression of miR-210-5p in the serum and CSF. (A). miRNAs in serum and CSF with CSF TPPA-negative or CSF TPPA-positive were investigated. (B). qPCR analysis of the relative quantitative expression of mir-210-5p in the CSF and serum isolated from syphilis patients with or without neurosyphilis. The first and second column represent CSF samples collected from CSF TPPA-negative and positive patients respectively. The third and fourth column data are serum samples from CSF TPPA-negative patients and CSF TPPA-positive patients respectively. Data are presented as means of relative abundance \pm SEM, $n>15$. CSF, cerebrospinal fluid; $C t$, cycle threshold; miRNA, microRNA; qPCR, quantitative PCR; TPPA, T pallidum particle agglutination.

\section{Statistics}

All statistical analyses were performed using GraphPad Prism (V.6.0, GraphPad Software). Results were considered to be statistically significant at $\mathrm{p}<0.05$.

\section{RESULTS}

\section{Identification of internal control miRNA for CSF and serum of TP-negative in humans}

miRNAs in the serum and CSF that are CSF $T$ pallidum particle agglutination (TPPA)-negative or CSF TPPA-positive were investigated (figure 1A). Out of 44 miRNAs examined in the qRT-PCR array, 32 miRNAs were detected with an average cycle threshold (Ct) of $>35$ and thus excluded from the study. Twelve miRNAs were detected to have below-average $\mathrm{Ct}$ value of 35, which showed uniformity at the expression level across these samples. All patients were serum TP-positive. The serum and CSF from patients with or without CSF TP infection were checked. The expression of miR-210-5p in the serum and CSF was stable (range 31.96-28.45, average 29.93 \pm 1.14 ) and basically consistent with the results of the experiment, and with no relationship with TP infection (figure 1B). Therefore, miR-210-5p was selected as the internal reference for subsequent analysis of miRNAs in the serum and CSF.

\section{Upregulated miRNA in CSF-positive samples compared with CSF-negative samples}

Compared with CSF-negative samples, in CSF-positive samples, miR-590-5p, miR-570-3p and miR-570-5p were upregulated in both the CSF and serum samples (figure $2 \mathrm{~A}, \mathrm{~B}, \mathrm{C}$ ). In the serum, miR-590-5p, miR-570-3p and miR-570-5p were significantly upregulated by $87.24 \%(\mathrm{p}<0.05), 533.92 \%(\mathrm{p}<0.01)$ and 95.02\% ( $<<0.01)$, respectively. miR-590-5p and miR-570-3p were highly upregulated in the CSF: $91.64 \%(\mathrm{p}<0.01)$ and $1137.7 \%(p<0.001)$. In addition, significant increase of miR-21-5p (nearly fourfold, $\mathrm{p}<0.05$ ) was observed only in the CSF sample, and not so in the serum sample, which displayed no statistical difference (figure 2D).

\section{Decreased miR-93-3p in CSF-positive samples compared with CSF-negative samples}

Compared with CSF-negative samples, miR-93-3p was decreased in both the CSF (down by 69.27\%, $\mathrm{p}<0.05$ ) and in the serum (down by $86.73 \%, \mathrm{p}<0.05$ ). The reduction was more pronounced in the serum (figure $3 \mathrm{~A}$ ).

\section{Altered miRNAs were not associated with neurosyphilis}

Although the expression of miR-27b-5p was higher in the CSF than in the serum, there is no difference between CSF-negative and CSF-positive samples, both in the CSF and in the serum (figure 3B). Furthermore, the expression of miR-16 and miR-23b-3p was less in the CSF than in the serum. There is also no difference between CSF-negative and CSF-positive samples in the CSF and in the serum (figure 3C, D).

\section{No difference in miRNAs}

For three miRNAs (miR-7-5p, miR-1307-5p and miR-203a-3p), no significant difference was detected, in the serum or in the CSF (figure 4).

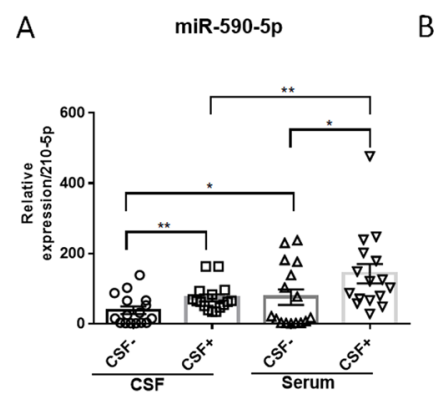

B

C

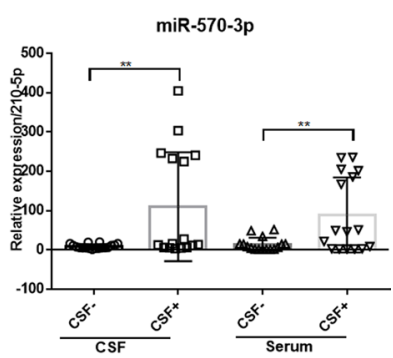

miR-570-5p

D

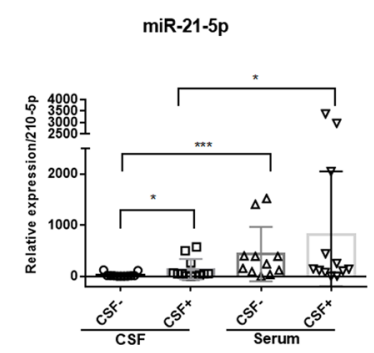

Figure 2 qPCR analysis of the relative quantitative expression of $(A)$ miR-590-5p, (B) miR-570-3p, (C) miR-570-5p and (D) miR-21-5p in the CSF and serum isolated from CSF TPPA-negative or CSF TPPA-positive patients. Data are presented as means of relative abundance \pm SEM, $\mathrm{n}>15$ per group. ${ }^{*} \mathrm{P}<0.05,{ }^{* *} \mathrm{P}<0.01,{ }^{* *} \mathrm{p}<0.001$. CSF, cerebrospinal fluid; qPCR, quantitative PCR. 
A

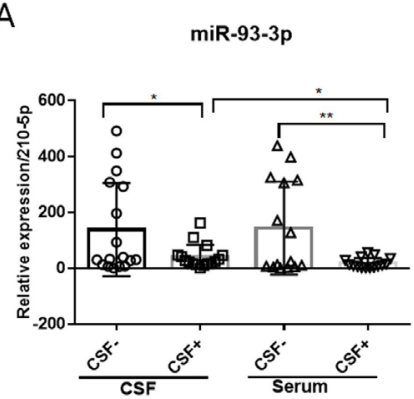

C

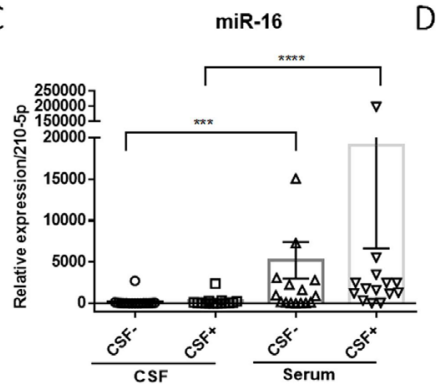

B

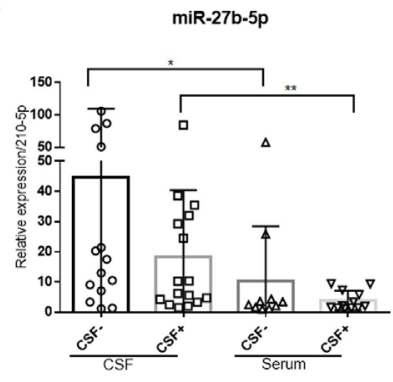

D

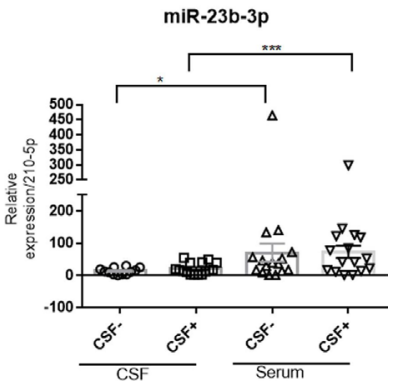

Figure 3 qPCR analysis of the relative quantitative expression of (A) miR-93-3p, (B) miR-27b-5p, (C) miR-16 and (D) miR-23b-3p in the CSF and serum isolated from CSF TPPA-negative or CSF TPPA-positive patients. Data are presented as means of relative abundance $\pm S E M$, $n>15$ per group. ${ }^{*} \mathrm{P}<0.05,{ }^{* *} \mathrm{p}<0.01,{ }^{* * *} \mathrm{p}<0.001,{ }^{* * *} \mathrm{p}<0.0001$. CSF, cerebrospinal fluid; $\mathrm{qPCR}$, quantitative PCR.

\section{DISCUSSION}

In recent years, miRNAs have received increasing attention and became hot spots in research. miRNAs are considered biological markers of multiple CNS tumours and diseases. The exosomes, as a transporter that can freely pass through the $\mathrm{BBB}$, contain miRNAs that are more direct and more researchable in the CNS.

In contrast to most mRNAs, detection of miRNAs in formalin-fixed tissue and CSF or other body fluid is more accurate and reliable. ${ }^{14}$ The aim of this study was to explore a predictive miRNA spectrum of circulatory blood to assess the possibility of nervous system infection condition, which will be of great help in clinical examination and treatment of neurosyphilis.

In previous studies on colorectal and breast cancer, it was found that miR-590-5p can target YAP1 and SOX2 to inhibit tumour occurrence, proliferation and metastasis. ${ }^{15}{ }^{16} \mathrm{~A}$ study of asthma discovered that the RNA-binding protein $\mathrm{HuR}$ is a direct target of miR-570-3p, which has implications for the expression of numerous other inflammatory mediators that $\mathrm{HuR}$ is known to regulate post-transcriptionally. ${ }^{17}$ Little research has been done

on miR-570-5p. miR-21-5p is considered to be an important tumour-related miRNA. It was found that miR-21-5p was associated with the growth and metastasis of multiple solid tumours. ${ }^{18}$ In a study investigating brain glioblastoma, miR-21-5p has been highly expressed. ${ }^{19}$ Moreover, a high expression in CSF in patients infected with Japanese encephalitis virus was detected. ${ }^{12}$ Similarly, in our study, we found that the expression of miR-21-5p was upregulated in the CSF of patients with neurosyphilis, which confirms that miR-21-5p is an miRNA closely related to CNS tumours and inflammation. Compared with CSF-negative samples, miR-590-5p,miR-570-3p and miR-570-5p were overexpressed in the CSF and serum in CSF-positive samples. miR-21-5p was overexpressed only in CSF. These results may suggest that these four miRNAs, especially miR-590-5p, may be positive regulators of TP-induced neuroinflammation.

$\mathrm{Xu}$ et al' ${ }^{20}$ study revealed differential expression of 17 miRNAs in the brain tissue from patients with HIV-associated neurocognitive disorders. They found a subset of upregulated miRNAs, including miR-93-3p. In our study, in CSF-positive samples, miR-93-3p was downregulated in the CSF and serum, when compared with CSF-negative samples. miR-590-5p and miR-93-3p are significantly upregulated or downregulated in the peripheral serum of serosin in the case of neurosyphilis. These miRNAs could be a tool for the diagnosis and observation of neurosyphilis in the future through peripheral blood.

The expression of the six miRNAs in the serum and the CSF (miR-7-5p, miR-1307-5p, miR-203a-3p, miR-16, miR-23b-3p and miR-27b-5p) was not affected in neurosyphilis.

The expression of miR-16 and miR-23b-3p was more prominent in the serum than in the CSF. The CSF expression of miR-27b-5p was higher than in the serum. Previous studies have found that miR-16, miR-23b-5p and miR-27b-5p have an effect on the proliferation, migration and invasion of malignant tumours. ${ }^{21}{ }^{22}$ Our findings suggested that miR-27b-5p is an miRNA that can pass through the BBB and can be expressed in the CNS higher than in the peripheral. Therefore it might be used as a drug carrier for treating diseases in the future such as neurosyphilis.

A possible limitation of our study might be that we did not include healthy individuals. We did test against patients with syphilis since this may be better to differentiate towards specific changes in the miRNA signature. Our purpose was to identify miRNAs that are specifically circulated in the CSF of patients with neurosyphilis.

In conclusion, our study underlines the importance of miRNAs in exosomes. Their presence in the CSF or in the serum could be either due to tissue damage or an inherent cellular mechanism for transporting specific miRNAs for cell-cell communication.
A

miR-7-5p

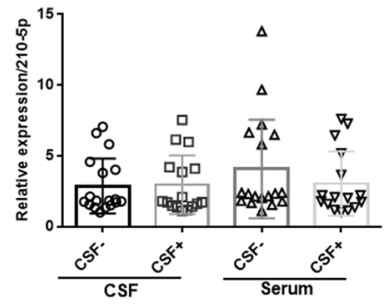

B

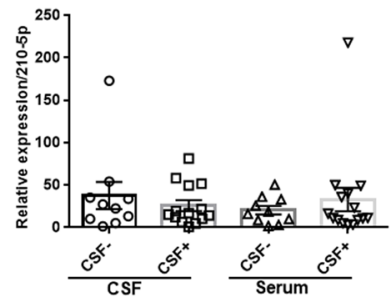

C miR-203a-3p

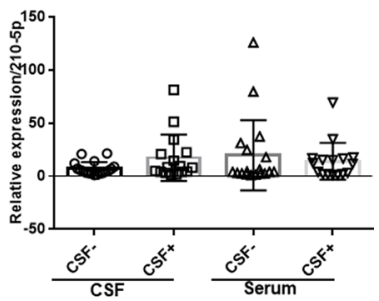

Figure 4 qPCR analysis of the relative quantitative expression of (A) miR-7-5p, (B) miR-1307-5p and (C) miR-203a-3p in the CSF and serum isolated from CSF TPPA-negative or CSF TPPA-positive patients. Data are presented as means of relative abundance \pm SEM, $n>15$ per group. CSF, cerebrospinal fluid; qPCR, quantitative PCR. 
Within infected cells, they could regulate various pathways contributing to the pathogenesis of neurosyphilis. Although higher sample size might be necessary, this work represents the first report of CSF and serum miRNAs in neurosyphilis and offers the basis for future investigation.

\section{Handling editor Khalil G Ghanem}

Acknowledgements The authors thank Professor Lilla Landeck for her critical revision of our manuscript.

Contributors $\mathrm{HC}$ and $\mathrm{YZ}$ collaborated in conducting and writing the manuscript. HC and Z-YW extracted the RNA from the serum and CSF. B-XY, W-FZ and T-TW collected samples from the patients, and B-XY performed the statistical analyses. $\mathrm{MZ}$ revised the manuscript before submission. X-YM designed and chose the main directions for data analysis and critically revised the manuscript.

Funding This study was supported by a grant from the National Natural Science Foundation of China (No 81773318).

Competing interests None declared.

Patient consent for publication Obtained.

Ethics approval The study was approved by the Ethics Committee of the Second Affiliated Hospital, Zhejiang University School of Medicine (IRB-2016023)

Provenance and peer review Not commissioned; externally peer reviewed.

\section{REFERENCES}

1 Marra CM. Neurosyphilis. Continuum 2015;21:1714-28.

2 Vlassov AV, Magdaleno S, Setterquist R, et al. Exosomes: current knowledge of their composition, biological functions, and diagnostic and therapeutic potentials. Biochim Biophys Acta 2012;1820:940-8.

3 EL Andaloussi S, Mäger I, Breakefield XO, et al. Extracellular vesicles: biology and emerging therapeutic opportunities. Nat Rev Drug Discov 2013;12:347-57.

4 Zhang Y, Liu D, Chen X, et al. Secreted monocytic miR-150 enhances targeted endothelial cell migration. Mol Cell 2010;39:133-44.

5 Zhuang X, Xiang X, Grizzle W, et al. Treatment of brain inflammatory diseases by delivering exosome encapsulated anti-inflammatory drugs from the nasal region to the brain. Mol Ther 2011:19:1769-79.
6 Sun D, Zhuang X, Xiang X, et al. A novel nanoparticle drug delivery system: the antiinflammatory activity of curcumin is enhanced when encapsulated in exosomes. $\mathrm{Mol}$ Ther 2010;18:1606-14.

7 Bolukbasi MF, Mizrak A, Ozdener GB, et al. miR-1289 and "Zipcode"-like Sequence Enrich mRNAs in Microvesicles. Mol Ther Nucleic Acids 2012;1:e10.

8 Frühbeis C, Fröhlich D, Krämer-Albers E-M. Emerging roles of exosomes in NeuronGlia communication. Front Physiol 2012;3.

9 De Smaele E, Ferretti E, Gulino A. MicroRNAs as biomarkers for CNS cancer and othe disorders. Brain Res 2010;1338:100-11.

10 Kalani A, Tyagi A, Tyagi N. Exosomes: mediators of neurodegeneration, neuroprotection and therapeutics. Mol Neurobiol 2014;49:590-600.

11 Skog J, Würdinger T, van Rijn S, et al. Glioblastoma microvesicles transport RNA and proteins that promote tumour growth and provide diagnostic biomarkers. Nat Cell Biol 2008;10:1470-6.

12 Goswami S, Banerjee A, Kumari B, et al. Differential expression and significance of circulating microRNAs in cerebrospinal fluid of acute encephalitis patients infected with Japanese encephalitis virus. Mol Neurobio/ 2017:54:1541-51.

13 Busk PK. A tool for design of primers for microRNA-specific quantitative RT-qPCR. BMC Bioinformatics 2014:15.

14 Mitchell PS, Parkin RK, Kroh EM, et al. Circulating microRNAs as stable blood-based markers for cancer detection. Proceedings of the National Academy of Sciences 2008:105:10513-8.

15 Ou C, Sun Z, Li X, et al. MiR-590-5p, a density-sensitive microRNA, inhibits tumorigenesis by targeting YAP1 in colorectal cancer. Cancer Lett 2017:399:53-63.

16 Zhou L, Zhao L-C, Jiang N, et al. MicroRNA miR-590-5p inhibits breast cancer cell stemness and metastasis by targeting Sox2. Eur Rev Med Pharmacol Sci 2017:21:87-94.

17 Roff AN, Craig TJ, August A, et al. MicroRNA-570-3p regulates HuR and cytokine expression in airway epithelial cells. Am J Clin Exp Immunol 2014;3:68-83.

18 Dai J, Lin Y, Duan Y, et al. Andrographolide inhibits angiogenesis by inhibiting the Mir21-5p/TIMP3 signaling pathway. Int J Bio/ Sci 2017;13:660-8.

19 de Mooij T, McCutcheon BA, Leontovich AA, et al. 336 Small RNA Sequencing of Glioblastoma Multiforme Extracellular Vesicles. Neurosurgery 2016:63(Suppl 1).

$20 \mathrm{Xu}$ Z, Asahchop EL, Branton WG, et al. MicroRNAs upregulated during HIV infection target peroxisome biogenesis factors: implications for virus biology, disease mechanisms and neuropathology. PLoS Pathog 2017;13:e1006360.

21 Zhou Y, Liu Y, Hu C, et al. MicroRNA-16 inhibits the proliferation, migration and invasion of glioma cells by targeting Sal-like protein 4. Int J Mol Med 2016:38:1768-76

22 Mazzio EA, Soliman KFA. HTP nutraceutical screening for histone deacetylase inhibitors and effects of HDACls on tumor-suppressing miRNAs by trichostatin $A$ and grapeseed (Vitis vinifera) in HeLa cells. Cancer Genomics Proteomics 2017;14:17-34. 